\title{
BERRY-ESSEEN BOUNDS FOR THE MULTI-DIMENSIONAL CENTRAL LIMIT THEOREM ${ }^{1}$
}

\author{
BY R. N. BHAT'TACHARYA
}

Communicated by P. R. Halmos, September 21, 1967

1. Introduction. Let $\left\{X_{n}\right\}$ be a sequence of independent and identically distributed random variables each with mean zero, variance unity, and finite absolute third moment $\beta_{3}$. Let $F_{n}$ denote the distribution function of $\left(X_{1}+\cdots+X_{n}\right) /(n)^{1 / 2}$. Berry [2] and Esseen [4] have proved that

(1) $\sup _{x \in R_{1}}\left|F_{n}(x)-\frac{1}{(2 \pi)^{1 / 2}} \int_{-\infty}^{x} e^{-y^{2} / 2} d y\right| \leqq c \beta_{3} / n^{1 / 2}, \quad n=1,2, \cdots$,

where $c$ is a universal constant. Consider now a sequence $\{X(n)$ $\left.=\left(X_{1}^{(n)}, \cdots, X_{k}^{(n)}\right)\right\}$ of independent and identically distributed random vectors in $R_{k}$ each with mean vector $(0, \cdots, 0)$ and covariance matrix $I$, the $k \times k$ identity matrix. If $P_{n}$ denotes the probability distribution of $\left(X^{(1)}+\cdots+X^{(n)}\right) / n^{1 / 2}$ and $\Phi$ is the standard $k$ dimensional normal distribution, then it is well known that $P_{n}$ converges weakly to $\Phi$ as $n \rightarrow \infty$. Bergström [1] has extended (1) to this case, assuming finiteness of absolute third moments of the components of $X^{(1)}$. Since weak convergence of a sequence $Q_{n}$ of probability measures to $\Phi$ means that $Q_{n}(B) \rightarrow \Phi(B)$ for every Borel set $B$ satisfying $\Phi(\partial B)=0, \partial B$ being the boundary of $B$, it seems natural to seek bounds of $\left|P_{n}(B)-\Phi(B)\right|$ for such sets $B$ (called $\Phi$-continuity sets). Let $Q$ be a class of Borel sets such that, whatever be the sequence $Q_{n}$ converging weakly to $\Phi, Q_{n}(B) \rightarrow \Phi(B)$ as $n \rightarrow \infty$ uniformly for all $B \in Q$. Such a class is called a $\Phi$-uniformity class. By a theorem of Billingsley and Topsoe [3], a class $Q$ is a $\Phi$-uniformity class if and only if $\sup \left\{\Phi(\partial B)^{\epsilon} ; B \in Q\right\} \downarrow 0$ as $\epsilon \downarrow 0$, where $(\partial B)^{\epsilon}$ is the $\epsilon$-neighborhood of $\partial B$. This leads one naturally to consider the class $a_{1}\left(d, \epsilon_{0}\right)$ of all Borel sets $B$ for which $\Phi(\partial B)^{\epsilon} \leqq d \epsilon$ for $0<\epsilon<\epsilon_{0}, d$ and $\epsilon_{0}$ being any two given positive constants. One may also consider the class $Q_{1}^{*}\left(d, \epsilon_{0}\right)$, which is the largest translation-invariant subclass of $a_{1}\left(d, \epsilon_{0}\right)$; this means that $B \in \mathbb{Q}_{1}^{*}\left(d, \epsilon_{0}\right)$ if and only if all translates of $B$ belong to $a_{1}\left(d, \epsilon_{0}\right)$.

1 The research for this work was supported in part by the Army Research Office, Office of Naval Research, and Air Force Office of Scientific Research by Contract No. Nonr-2121(23), NR 343-043. 
2. Results. We shall write $\beta_{s}=\sum_{i=1}^{k} E\left|X_{i}^{(1)}\right|^{s}$ for $s>0$. Also $c$ 's will denote constants. For example, $c_{1}(k, \delta), c_{2}(k)$, and $c_{4}$ will stand for a constant depending only on $k$ and $\delta$, a constant depending on $k$ alone, and a universal constant, respectively.

TheOREM 1. Suppose $\beta_{3+\delta}<\infty$ for some $\delta>0$. Then, for all $n$, $\sup \left|P_{n}(B)-\Phi(B)\right|$

$$
\leqq n^{-1 / 2}\left\{c_{1}(k, \delta) \beta_{3+\delta}^{3(1+\delta) /(3+\delta)}+\left[c_{2}(k) d+c_{8}(k) / \epsilon_{0}\right] \beta_{3+\delta}^{3 /(3+\delta)}\right\},
$$

where the supremum extends over all $B$ in $Q_{1}^{*}\left(d, \epsilon_{0}\right)$.

We shall state two applications of Theorem 1.

EXAMPLE 1. Let $\mathcal{e}$ be the class of all measurable convex sets in $R_{k}$. It follows from certain results of Ranga Rao [5] that $\mathcal{C} \subset Q_{1}^{*}\left(d(k), \epsilon_{0}\right)$ for every $\epsilon_{0}>0, d(k)$ being an appropriate constant depending on $k$. Hence

$$
\sup _{C \in \mathcal{C}}\left|P_{n}(C)-\Phi(C)\right| \leqq n^{-1 / 2}\left\{c_{1}(k, \delta) \beta_{3+\delta}^{3(1+\dot{o}) /(3+\delta)}+c_{2}(k) d(k) \beta_{3+\delta}^{8 /(3+\delta)}\right\}
$$

for all $n$. This is an improvement on a result of Ranga Rao [6].

EXAMPLE 2. Let $F(l)$ be the class of all measurable sets in $R_{2}$ each of whose boundaries is contained in a rectifiable curve of length not exceeding $l$. It may be shown (cf. [3]) that $\mathfrak{F}(l) \subset \mathfrak{Q}_{1}^{*}(4 \pi l+8 \pi, 1)$. Hence Theorem 1 applies. In fact, in this case it suffices to assume that $\beta_{3}<\infty$, so that we have

$$
\sup _{F \in \mathcal{F}_{(l)}}\left|P_{n}(F)-\Phi(F)\right| \leqq n^{-1 / 2}\left\{\left(c_{4} l^{2}+c_{5} l+c_{8}\right) \beta_{3}\right\}, \quad n=1,2, \cdots .
$$

Theorem 2. Suppose $\beta_{3+\delta}<\infty$ for some $\delta>0$. Then, for all $n$,

$$
\begin{aligned}
\sup \mid P_{n}(B) & -\Phi(B) \mid \\
& \leqq n^{-1 / 2}\left\{c_{7}(k, \delta) \beta_{3+\delta}^{6 /(3+\delta)}+c_{8}(k)\left[d+1 / \epsilon_{0}\right] \beta_{8+\delta}^{3 /(3+\delta)} \log (n+1)\right\},
\end{aligned}
$$

where the supremum extends over all $B$ in $Q_{1}\left(d, \epsilon_{0}\right)$.

The methods used in proving Theorems 1 and 2 enable one to obtain bounds for general $\Phi$-uniformity classes, and, in particular, for any $\Phi$-continuity set.

An asymptotic expansion holds for the class $a_{1}\left(d, \epsilon_{0}\right)$ under the assumption that

$$
\limsup _{|t| \rightarrow \infty}|f(t)|<1
$$


where $f$ is the characteristic function of $X^{(1)}$. If $\beta_{8}<\infty$ for some integer $s \geqq 3$, then $P_{n}(B)$ may be estimated by this expansion with an error $O\left(n^{-(8-2) / 2} \cdot[\log n]^{k / 2}\right)$ uniformly for all $B \in Q_{1}\left(d, \epsilon_{0}\right)$.

Extensions. Theorems 1 and 2 may be extended to the following cases: (1) $\left\{X^{(n)}\right\}$ is not identically distributed, but $\sup _{n} \sum_{i=1}^{k} E\left|X_{i}^{(n)}\right|^{3+\delta}<\infty$ for some $\delta>0$; (2) $\left\{X^{(n)}\right\}$ has a common nonsingular covariance matrix perhaps different from $I$.

In proving Theorem 1 we look at the convolution $\left(P_{n}-\Phi\right) * \mathrm{I}_{n}$, where $\Gamma_{n}$ is a probability measure having a characteristic function which vanishes everywhere outside a sphere, and $\Gamma_{n}$ converges weakly to the probability measure degenerate at $(0, \cdots, 0)$. Theorem 2 is obtained by sharpening a technique of Esseen [4] and Ranga Rao [5].

The details and proofs of these results, which are part of the author's doctoral dissertation, submitted to the University of Chicago, will appear elsewhere.

Acknowledgement. I am extremely grateful to Professor Patrick Billingsley for guidance and encouragement, and, in particular, for suggesting the above investigation.

\section{REFERENCES}

1. H. Bergström, On the central limit theorem in $R_{k}, k>1$, Skand. Aktuarietidskr. 28 (1945), 106-127.

2. A. C. Berry, On the accuracy of Gaussian approximation to the sum of independent variates, Trans. Amer. Math. Soc. 49 (1941), 122-136.

3. P. Billingsley and F. Topsoe, Uniformity in weak convergence, $Z$. Wahrscheinlichkeitstheorie und Verw. Gebiete. 7 (1967), p. 1-16.

4. C. G. Esseen, Fourier analysis of distribution functions: A mathematical study of the Laplace-Gaussian law, Acta Math. 77 (1945), 1-125.

5. R. Ranga Rao, Some problems in probability theory, Ph.D. thesis, Calcutta University, 1960.

6. - On the central limit theorem in $R_{k}$, Bull. Amer. Math. Soc. 67 (1961), $359-361$.

University of Chicago 